\title{
COMPRESSION OF ECAPED TITANIUM MICRO-PILLARS FOR TWO PRINCIPAL ORIENTATIONS
}

\author{
David Vokoun*, Jan Mañák, Karel Tesař, Stanislav Habr
}

\author{
Czech Academy of Science, Institute of Physics, Na Slovance 1999/2, Prague, Czech Republic \\ * corresponding author: vokoun@fzu.cz
}

\begin{abstract}
The thermomechanical processing by equal-channel angular pressing (ECAP) is used for certain metals and alloys in order to make their structure fine and to increase material strength. In the previous study done at our institute, grade 2 titanium was successfully processed using four consecutive route A passes via a $90^{\circ} \mathrm{ECAP}$ die with high backpressure at room temperature. Orientation dependence of compressive and tensile loading of ECAPed titanium samples was demonstrated at macro-scale. However, scarce attention has been paid so far to the mechanical behavior of ECAPed titanium samples at micro-scale. In the present study, compression experiments on titanium micropillars, fabricated using focused ion beam, are carried out for two main directions in respect to preceding ECAP pressing (insert and extrusion directions). The purpose of this study is to discuss the orientation dependence of mechanical response during compression of the as-ECAPed titanium micro-pillars.
\end{abstract}

KEYWORDS: ECAP, micro-pillar, titanium.

\section{INTRODUCTION}

Titanium is a light-weight material with hexagonal structure at room temperature. Due to high specific strength (strength-to-weight ratio), titanium and titanium based alloys are used in car- and aerospaceindustries. In addition, titanium and its oxides are biocompatible. Sound biocompatibility together with high resistance to the onset of localized corrosion make titanium a good candidate for biomedical applications. Furthermore, fabrication of metalic micro-parts has been receiving much attention in recent years due to the rapid development of micro-electro-mechanical systems (MEMS). Therefore, understanding of mechanical properties of various metalic materials including titanium at micrometric level is of importance. It only makes sense to produce and mechanically test microparts which are either monocrystalline with a fixed relation between the crystallographic and geometric axes [1] or which contain submicron grains (grains which are small enough to guarantee reproducibility of mechanical tests carried out on arbitrary two microsamples of identical dimensions). However, grains of titanium samples produced in a conventional way are large and their refinement requires special methods.

The small tensile strength of titanium (commercially pure, grade 2 titanium, has tensile strength only $345 \mathrm{MPa}$ 2]) can be increased either through alloying [3, 4] (a high chance of decreasing or losing biocompatibility), or through severe plastic deformation (SPD) processes used for grain refinement. The increase of tensile strength in various metals and alloys through ECAP (Equal Channel Angular Pressing) and other SPD methods is generally known, e.g. the authors of 5 showed an increase of tensile strength of ECAPed commercially pure (CP) titanium due to the ECAP process up to $1000 \mathrm{MPa}$ (true stress). ECAP is a wide-spread SPD technique [6] used also for titanium and titanium based alloys. During an ECAP procedure, samples (billets) are heavily deformed with strains close to or exceeding $100 \%$ (depending on the used die geometry and the number of passes) due to repeated pressing a metalic billet through a system of two channels, axes of which intersect under an abrupt angle, usually $120^{\circ}$ or $90^{\circ}$. A great number of studies, so far published, describe preparation of ECAPed titanium samples and evaluate microstructure, various physical and mainly mechanical properties of ECAPed titanium samples in dependence on the ECAP parameters [5, 7-11].

The key ECAP parameters are related first to the orientation of a billet upon the repeated insertion in a die in respect to the previous passing of the billet through the die (routes A, Ba, Bc, and C [6] ). The ECAP parameters are also related to the die geometry and the billet temperature. Angle $\varphi$ (channel angle) is the abrupt angle between the two die channels and angle $\psi$ (corner angle) represents the outer arc of curvature where the two channels intersect [6]. The other ECAP parameters are e.g. number of the passes through a die, pressing speed, values of backpressure, pressing temperature, etc.

Room-temperature ECAP processing is not so common for titanium although it brings the advantage of enhanced grain refinement due to the suppressed grain structure recovery. Room-temperature ECAP processing using channel angle $90^{\circ}$ and less with CP grade 2 titanium is still challenging [5] due to material cracking after the first or second pass of the material through the die. In Ref. [5], room-temperature ECAP processing is described and later used in our study in exactly the same way with the same titanium alloy (identical chemical composition). 


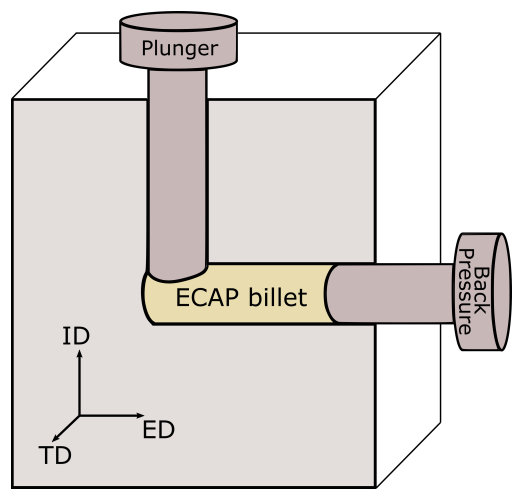

Figure 1. Scheme of the ECAP die with the coordinate system. The abbreviations ID, ED and TD represent the insert, extrusion and transverse directions, respectively.

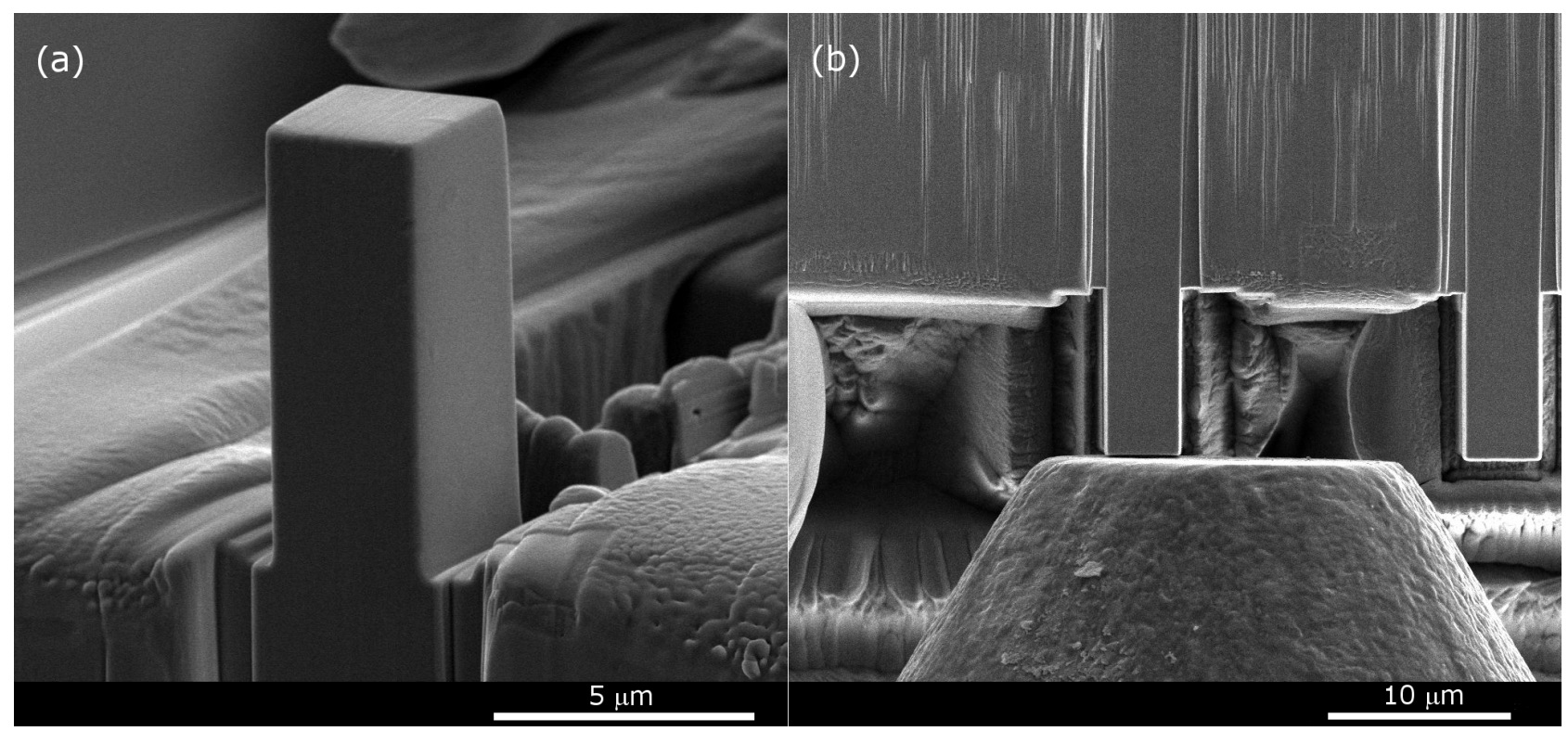

Figure 2. The SEM image of a micro-pillar (a) and the diamond tip approaching one of the fabricated micro-pillars (b).

The purpose of the present work is to extend the previous work [5] in relation to compression experiments at room temperature. To our knowledge, so far no work on compression of ECAPed titanium micropillars has been done. However, mechanical testing on micro-pillars of other ECAPed metals and alloys has been performed recently [12, 13. The advantage of our work is the availability of compressive tests on ECAPed titanium at both macro- and micro-scale and the possibility of comparison.

\section{SAMPLES}

A cube, $3 \mathrm{~mm} \times 3 \mathrm{~mm} \times 3 \mathrm{~mm}$, made of ECAPed titanium was cut out (using wire electro-discharge machining) in such a way that each cube face was perpendicular to one of the principal directions - insert direction (ID), extrusion direction (ED) and transverse direction (TD) (See Fig. 1). The micro-pillars, each in the shape of a rectangular prism with a square base (See Fig. 2a), were micro-fabricated and observed using an FEI Quanta 3D Dual-Beam SEM/FIB (scan- ning electron microscope/focused ion beam) system (FEI, Hillsboro, USA) with a Ga+ ion source operated at $30 \mathrm{keV}$ with various currents using automated milling script. Each micro-pillars' face was again perpendicular to one of the principal directions (ID, ED, and TD). As for dimensions of the micro-pillars, their height and width were approximately $8.5 \mu \mathrm{m}$ and $3.5 \mu \mathrm{m}$, respectively. The micro-pillars were of two types denoted as ID and ED micro-pillars. ID $\{\mathrm{ED}\}$ micro-pillars had their long axis parallel to ID $\{\mathrm{ED}\}$ direction, respectively. No micro-samples with pillar's long axis parallel to TD direction were prepared since previous studies showed almost identical mechanical characteristics in ID and TD directions. It was checked that each micro-pillar contained grains small enough not to limit reproducibility of the following compression tests.

There were three ID and four ED micro-pillars fabricated; together, seven micro-pillars. For each micropillar just one force-displacement curve was measured and converted into stress-strain curve. However not all 
stress-strain data were used. Most stress-strain curves were excluded because of a misalignment during the compression.

\section{Methods}

The ECAP die with a channels' diameter of $11 \mathrm{~mm}$ and the channel and corner angles equal to $90^{\circ}$ and $0^{\circ}$, respectively, was used. Back pressure regulated between $270 \mathrm{MPa}$ and $590 \mathrm{MPa}$ to maintain an average plunger pressure of $2800 \mathrm{MPa}$ was applied during the ECAP process. The inserted titanium rod-like sample, $35 \mathrm{~mm}$ long, was encapsulated in a lubricant-treated thin steel container in order to eliminate frictional forces. The details of the ECAP process can be found in [5]. The ECAP process was carried out at room temperature using route $\mathrm{A}$. The number of passes of the billet through the die was four. The in situ compression tests were performed at room temperature using a Hysitron PI 85 SEM PicoIndenter (Hysitron, Minneapolis, USA) with a $20 \mu \mathrm{m}$ flat-punch diamond tip. Figure $2 \mathrm{p}$ shows the diamond tip approaching one of the fabricated micro-pillars. The true strain rate was around $5.2 \times 10^{-4} \mathrm{~s}^{-1}$. The micro-pillars were compressed up to about $10 \%$. A special care was given to the alignment of the loading and micro-pillars' long axes during loading. The loading data with an obvious difference in Young's moduli at the onset of loading and unloading were excluded.

The grain morphology at the micro-pillar longitudinal sections were acquired using the FEI Quanta $3 \mathrm{D}$ equipped with an EDAX Hikari EBSD (electron backscatter diffraction) detector (EDAX, New Jersey, USA) and analyzed with a help of EDAX OIM Analysis v8 software.

\section{Results And Discussion}

The grain morphology of the micro-pillars (not shown here) corresponds to the micro-structure of the ECAPed titanium samples studied in [5]. Herein, it is worth mentioning that the mean grain size determined in [5] using an X-ray diffraction (XRD) (crystallite size calculated from line broadening of the XRD peaks) was $50 \mathrm{~nm}$ and the prevailing grain size determined in [5] using a transmission electron microscope was in the range $50 \mathrm{~nm}-300 \mathrm{~nm}$. The EBSD map of an ECAPed Ti sample processed identically as the present sample can be found in [14]. As of the present micro-samples, there are regions of recrystallized grains with a mean grain size of $200 \mathrm{~nm}$ and regions of non-recrystallized grains. The grain size of the largest non-recrystallized grains does not exceed $3 \mu \mathrm{m}$. The micro-samples contain only a few large grains and these grains still contain regions with various crystallographic orientations. In addition, an arbitrary micro-pillar cross-section area $(3.5 \mu \mathrm{m} \times 3.5 \mu \mathrm{m})$ contains at least five grains. Low-angle grain boundaries prevail among the noncrystallized grains. Furthermore, it can be assumed that high deformation energy is accumulated in the regions of non-recrystallized grains. Figure 3 shows the diagram of true compression stress versus true strain for ID and ED micro-pillars, ID and ED macrosamples and titanium bulk sample. In Fig. 3, the stress-strain data belonging to compression loading of ECAPed titanium samples denoted as ID and ED macro-samples and titanium macro-sample with no ECAP treatment denoted as Ti bulk (coarse-grained sample) were taken from our previous study [5]. The ID, ED and Ti-bulk macro-samples had a shape of a cylinder with a diameter of $3 \mathrm{~mm}$, a height of $6 \mathrm{~mm}$ and their rotational axes along ID, ED and LD directions, respectively. All the samples were mechanically tested at room temperature.

In [5], it is shown that at macroscopic level, mechanical properties of ECAPed titanium are anisotropic. The maximum compression stress in ID direction is about $24 \%$ higher than the maximum compression stress in ED direction (See Fig. 3.

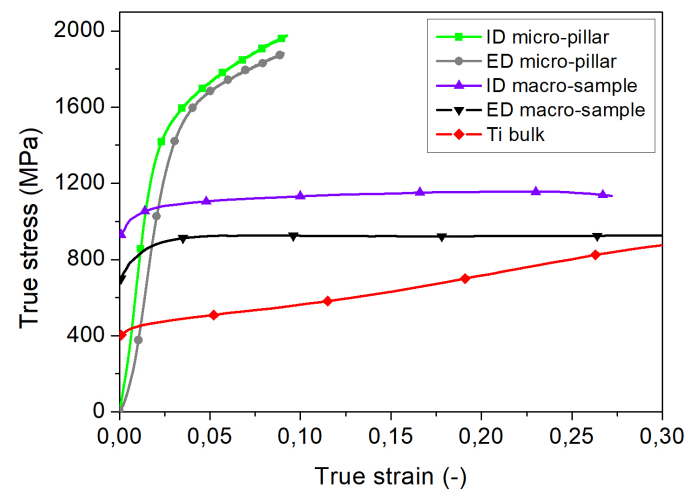

Figure 3. The diagram true compression stress versus true strain for ID and ED micro-pillars, ID and ED macro-samples and titanium bulk sample.

At microscopic level, in case of ID and ED micropillars, maximum stress in ID micro-pillar is still greater than maximum stress in ED micro-pillar but the stress ratio, $\left(\sigma_{\mathrm{ID}}\right)_{\max } /\left(\sigma_{\mathrm{ED}}\right)_{\max }$, is close to 1 . There is a question whether the micro-samples are less anisotropic (in compression) than the macro-samples. However, at this stage of our study, it is not possible to make any conclusion. Stress-strain data for more micro-pillars are necessary in further research. If it turns out that there really is less compression anisotropy in the micro-pillars compared to the macrosamples, then a potential explanation might be as follows: The effect of anisotropy in compression, i.e. yield strength depends on the compression direction, is likely due to anisotropic distribution of dislocations in the ECAPed samples. If the size of the Ti ECAPed samples decreases, as in the present study, to units of microns, then the plastic deformation (during compression) is associated with the creation and movement of newly formed dislocations, rather than with motion and interactions of already existing dislocations (as it is in bulk samples) [15]. This means there is a weaker 
effect of the existing anisotropic distribution of dislocations. This might be a reason for the potentially less significant effect of compression anisotropy in the micro-pillars, compared to the macro-samples.

Another distinct phenomenon in Fig. 3 is the samplesize effect, obvious when comparing the true stress true strain curves belonging to the micro-pillars and the ECAPed titanium macro-samples. It is assumed that the mean grain size of the compared samples is approximately identical.

$\mathrm{Xu}$ et al. [16] observed the sample-size effect for pure aluminum in the form of softening behavior (negative work-hardening rates) and a non-significant yield stress change with a reduction of the specimen size. However, for the fixed small grain sizes, the authors did not test sample dimensions for which the sample-size effect is significant i.e. when the sample size decreases to dimensions comparable to about ten times the average grain size. In addition, the crystal structure of aluminum is cubic whereas the crystal structure of titanium is hexagonal at room temperature. In the present study, both yield stress and the work-hardening rate increased remarkably with the reduction of sample dimensions to only units of microns. These observations are perfectly consistent with compression tests done on ECAPed Mg-5 wt.\% Al micro-fabricated into a shape of micro-pillars [17. The sample-size effect observed in the present study is based on the dislocation starvation theory [15] and the effect of grains close to the sample's surface since the volume ratio of surface grains increases with the sample size decrease. The theoretical background of the observed sample-size effect might be found also in [18.

\section{Conclusions}

ECAPed titanium (grade 2) is successfully reproduced at room temperature (route A, four passes) using an ECAP-Back-Pressure die with a channels' diameter of $11 \mathrm{~mm}$ and the channel and corner angles equal to $90^{\circ}$ and $0^{\circ}$, respectively. A concern was raised that ECAPed titanium micro-samples in compression might be less anisotropic than the macrosamples. Further study is necessary to clarify this concern. Furthermore, comparing the compression stress - strain curves for ECAPed titanium microsamples and macro-samples, both yield stress and the work-hardening rate increase remarkably with the reduction of sample dimensions.

\section{ACKNOWLEDGEMENTS}

Support of the work under the project GA17-05360S is acknowledged.

\section{REFERENCES}

[1] J. Maňák, D. Vokoun. Microbending experiments on pure magnesium with nonbasal slip orientation. Materials 11(8):1434, 2018. DOI:10.3390/ma11081434
[2] M. Niinomi. Mechanical properties of biomedical titanium alloys. Materials Science and Engineering: A 243(1-2):231-236, 1998. DOI:10.1016/s0921-5093(97)00806-x.

[3] E. Ezugwu, Z. Wang. Titanium alloys and their machinability - a review. Journal of Materials Processing Technology 68(3):262-274, 1997. DOI:10.1016/s0924-0136(96)00030-1.

[4] Y. Okazaki, Y. Ito, A. Ito, T. Tateishi. Effect of alloying elements on mechanical properties of titanium alloys for medical implants. Materials Transactions, JIM 34(12):1217-1222, 1993. DOI:10.2320/matertrans1989.34.1217

[5] A. Jäger, V. Gärtnerova, K. Tesař. Microstructure and anisotropy of the mechanical properties in commercially pure titanium after equal channel angular pressing with back pressure at room temperature. Materials Science and Engineering: A 644:114-120, 2015. DOI:10.1016/j.msea.2015.07.038

[6] R. Z. Valiev, T. G. Langdon. Principles of equal-channel angular pressing as a processing tool for grain refinement. Progress in Materials Science 51(7):881-981, 2006. DOI:10.1016/j.pmatsci.2006.02.003

[7] C. S. Meredith, A. S. Khan. The microstructural evolution and thermo-mechanical behavior of UFG ti processed via equal channel angular pressing. Journal of Materials Processing Technology 219:257-270, 2015. DOI:10.1016/j.jmatprotec.2014.12.024.

[8] X. Zhao, X. Yang, X. Liu, et al. Processing of commercial purity titanium by ECAP using a 90 degrees die at room temperature. Materials Science and Engineering: A 607:482-489, 2014. DOI:10.1016/j.msea.2014.04.014.

[9] D. Shin, I. Kim, J. Kim, et al. Microstructure development during equal-channel angular pressing of titanium. Acta Materialia 51(4):983-996, 2003. DOI:10.1016/s1359-6454(02)00501-3.

[10] M. Hoseini, M. H. Pourian, F. Bridier, et al. Thermal stability and annealing behaviour of ultrafine grained commercially pure titanium. Materials Science and Engineering: A 532:58-63, 2012. DOI:10.1016/j.msea.2011.10.062.

[11] H. Mora-Sanchez, I. Sabirov, M. A. Monclus, et al. Ultra-fine grained pure titanium for biomedical applications. Materials Technology 31(13):756-771, 2016. DOI:10.1080/10667857.2016.1238131

[12] C. Howard, D. Frazer, A. Lupinacci, et al. Investigation of specimen size effects by in-situ microcompression of equal channel angular pressed copper. Materials Science and Engineering: $A$ 649:104-113, 2016. DOI:10.1016/j.msea.2015.09.110.

[13] Z. Liao, M. Polyakov, O. G. Diaz, et al. Grain refinement mechanism of nickel-based superalloy by severe plastic deformation - mechanical machining case. Acta Materialia 180:2-14, 2019. DOI:10.1016/j.actamat.2019.08.059

[14] H. Sopha, K. Tesar, P. Knotek, et al. TiO2 nanotubes grown on ti substrates with different microstructure. Materials Research Bulletin 103:197-204, 2018. DOI:10.1016/j.materresbull.2018.03.036 
[15] C. Frick, B. Clark, S. Orso, et al. Size effect on strength and strain hardening of small-scale [111] nickel compression pillars. Materials Science and Engineering: A 489(1-2):319-329, 2008. DOI:10.1016/j.msea.2007.12.038

[16] J. Xu, X. Zhu, D. Shan, et al. Effect of grain size and specimen dimensions on micro-forming of high purity aluminum. Materials Science and Engineering: A 646:207-217, 2015. DOI:10.1016/j.msea.2015.08.060.
[17] H. Diao, C. Yan, J. Bell, et al. Deformation behaviour of nanocrystalline mg-al alloys during nanoindentation. In 2010 3rd International Nanoelectronics Conference (INEC). IEEE, 2010. DOI:10.1109/inec.2010.5424788

[18] V. Kafka, D. Vokoun. Nanowires: diameter-dependent strength criterion. IOP Conference Series: Materials Science and Engineering 504:012110, 2019. DOI:10.1088/1757-899x/504/1/012110 